\title{
The robotized stand for the diagnosing compression refrigerating machines
}

\author{
Sergey Petrosov ${ }^{1}$, Michael Lemeshko ${ }^{1, *}$, and Alexander Kozhemyachenko ${ }^{1}$ \\ ${ }^{1}$ Don state technical university (branch) Shakhty, Rostov region, Russia
}

\begin{abstract}
The process of creation and improvement of small refrigerating machines, the way of increasing their energy efficiency and reducing costs should be provided with reliable means and effective technologies of technical diagnosing these machines. One of the methods for achieving this goal is the creation of robotic research complexes. The authors, using the example of the automated research stand, show a possible variant of solving research problems when the researcher needs to make a measurement program, including a program of varying experimental conditions. Long-term tests with recording measurement results are performed in automatic mode without the operator-researcher's participation. The robotic stand usually replaces the research operator, and ensures the effective results of long-term studies as they can be carried out more qualitatively and deeply, with simultaneous evaluation of results and adjustments of the measurement plans. The scheme and description of the developed stand are given. The significant advantages of a robotic stand are shown in comparison with the known analogous means of technical diagnostics of small refrigerating machines.
\end{abstract}

The process of creating new machines, tools and equipment, their improvement determined the tasks of scientific justification of new ideas, new design and technological solutions. The operation and improvement of small refrigerating machines, the process of modernization are also impossible without scientific, theoretical and experimental research methods and new testing technologies.

One of the directions of assessing the quality of new refrigerating machines, testing new ideas is to test such machines on diagnostic stands. Of course, theoretical research can predict the expected effects and results of new technical solutions to improve the performance of small refrigerators. However, relatively complex mass-exchange processes, specific cycles of compression operation in refrigerating machines are known to be investigated by means of experimental methods[1].

To solve the problems of evaluating the performance efficiency of small refrigerators, different approaches and criteria are used. For example, a well known method is used for determining deviations from the normal functioning modes of refrigerating machines including domestic refrigerators [2]. In this method, the signatures based on a plurality of subsystem sensors of the refrigerator are taken into account, and the efficiency of the refrigerator is estimated by means of comparing the reference values with the measured

${ }^{*}$ Corresponding author: lem-mikhail@ya.ru 
indicators. This method of evaluating new developments is unacceptable, since it does not eliminate external factors that can greatly distort the evaluation of the refrigerating machines efficiency.

Stands and devices for diagnostics of small refrigerators (SR) are well known. For example, the stand is used for expeditious determination of technical condition of household refrigerators SH-2[3]. The stand can ensure the check of temperature conditions in cameras, and evaluate the current power consumption. However, the influence of air temperature changes affecting the indicators is ignored in such stands.

Also systems and the equipment for diagnostics and assessment of the efficiency of refrigerators in general or their separate subsystems are known. These are specialized stationary stands with a heat-insulated camera and a calorimeter.

For example, there is a stand for testing the hermetic unit [4] in the figure 1.

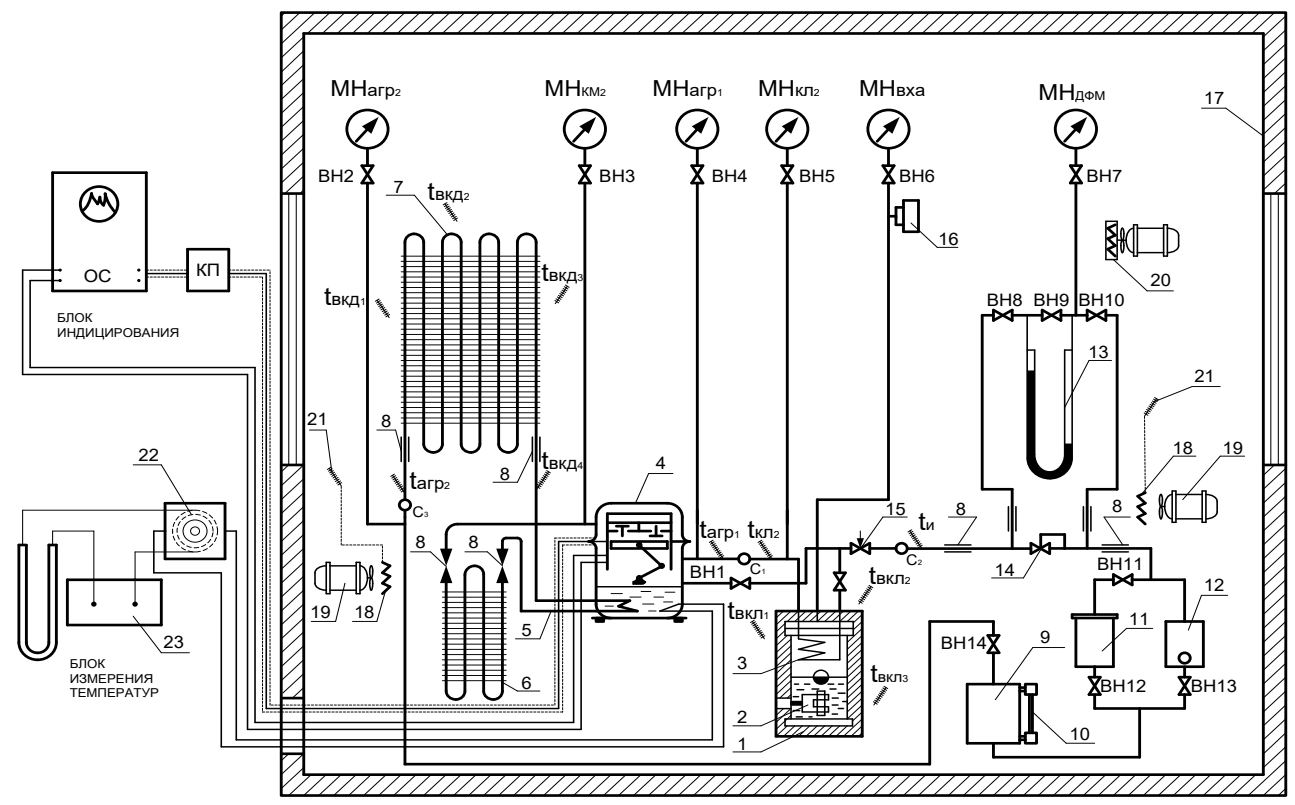

Fig. 1. Schematic diagram of the calorimetric stand of testing hermetic units of household refrigerators and freezes

1 - electric calorimeter; 2 - electric heating element; 3 - coil evaporator; 4 - compressor; 5 - coiling oil cooler; 6 capacitor; 7 - condenser; 8 - connecting coupling; 9 - receiver; 10 - measured glass; 11 technological filter dehumidifier; 12 - the device for defining oil concentration; 13 - differential mercury manometer; 14 - the device for regulating the pass ability of the liquid line; 15 - the regulating valve; 16 - pressure relay; 17 - the heat-insulated camera; 18 - electric heater; 19 - fan; 20 - condenser; 21 - contact thermometer

The approach to diagnose small refrigerators is based on refrigerating capacity as integrated indicator [5] which is realized in the stand. It is also possible to use this stand for diagnostics of local subsystems of the refrigerator [6].

Integrated operation indicator of the studied machine is the specific energy consumption. Another integrated criterion of refrigerating cycle efficiency is the temperature stability in cameras [7]. More precisely: temperatures in cameras should not be beyond the temperature requirements of the products' storage conditions.

Such stands ensure the opportunity to conduct researches in thermally closed system under stable air temperatures. The main disadvantage of the considered stands and other similar stands with the calorimeter and heat-insulated camera is the obligatory operator's participation during the process of taking measurements, the bulkiness of the stand, the 
time duration of tests, and manual control of measurements by means of rotating valves, the process of removal the indicators on manometers and thermometers scales. At the same time measurements may have rather high errors. Temperature in the heat-insulated camera is regulated by the contact thermometer which doesn't provide the accuracy of temperature adjustments and gives essential errors in process of measurements.

Also other stands and methods of diagnostics of small refrigerators are known. For example, designs of stands and technology of diagnostics used for the following patents $[8,9,10]$. The analysis of the known technologies and stands for diagnosing heat power efficiency of small refrigerators allowed defining their general features -the necessity of the operator's presence during rather long tests, the complexity of organizing exact identity of test conditions, rather low reliability and accuracy of research results.

It is obvious to develop a new modern automated stand to improve small refrigerating machines and effectively investigate refrigeration processes.

The authors have developed the concept of the robotic stand for diagnosing small refrigerators and for researching heat power characteristics at various air temperatures [11].

The created robotic stand includes the heat-insulated camera, the regulation system, control and sub-control of air temperature in the camera. This system allows establishing and maintaining temperature in the heat-insulated cameras programmatically.

The system can give orders for taking measurements after the end of each transition process caused by transition from one air temperature indicators to another. The stand includes a group of sensors for measuring operation indicators of small refrigerating machines.

The scheme of the developed robotic stand is given in the figure 2.

The stand consists of the heat-insulated camera 1, the studied refrigerator 3 having two cameras - low-temperature and the cooling camera, the condenser 8 , the compressor 11 ; conditioner 4, thermal generators 5; the block of planning measurements 12 , the block of programming temperature changes 13 in the heat-insulated camera 1 , the controller of estimating the process of measurements 14 , cable lines between sensors and the block of planning measurements, cable lines between temperature sensors 6,9 , thermal generators 5 , the conditioner 4 and the block of programming temperature changes in the heat-insulated camera 14.

The stand can include other measuring sensors, for example, for measuring humidity in the refrigerator cameras, and/or for estimating noise characteristics of the compressor and other sensors for measuring indicators of refrigerators' operation.

Before turning on of the stand and carrying out measurements some preparatory operations are to be carried out.

The small refrigerator is placed in the heat-insulated camera on the support raised over floor level on rated level according to the standard [11]; temperature sensors, pressure sensors, sensors of power consumption are placed in the studied cameras and subsystems of the studied SRM.

The quantity and type of sensors in groups and their places before measurements are defined by the research problems, for example, for researching the air temperature influence on heat power indicators of small refrigerators, it is expedient to place temperature sensors in the cooled cameras and also to place sensors of power consumption on the compressor and the fan.

After preparatory operations being over the stand is turned on. Programming measurement plan is carried out, the list of temperatures is programmed ( $\mathrm{t}$ cam ${ }^{\wedge} \mathrm{n}$ where $\mathrm{n}=1,2,3, \ldots)$ in the heat-insulated camera and measurements of the refrigerator indicators are automatically taken, for different temperature indicators $\mathrm{t} \_$cam ${ }^{\wedge} 1=25^{\circ} \mathrm{C}$, by $\mathrm{t} \_$cam ${ }^{\wedge} 2=30^{\circ} \mathrm{C}$, t_cam ${ }^{\wedge} 3=32^{\circ} \mathrm{C}$, t_cam ${ }^{\wedge} 4=38^{\circ} \mathrm{C}$. 


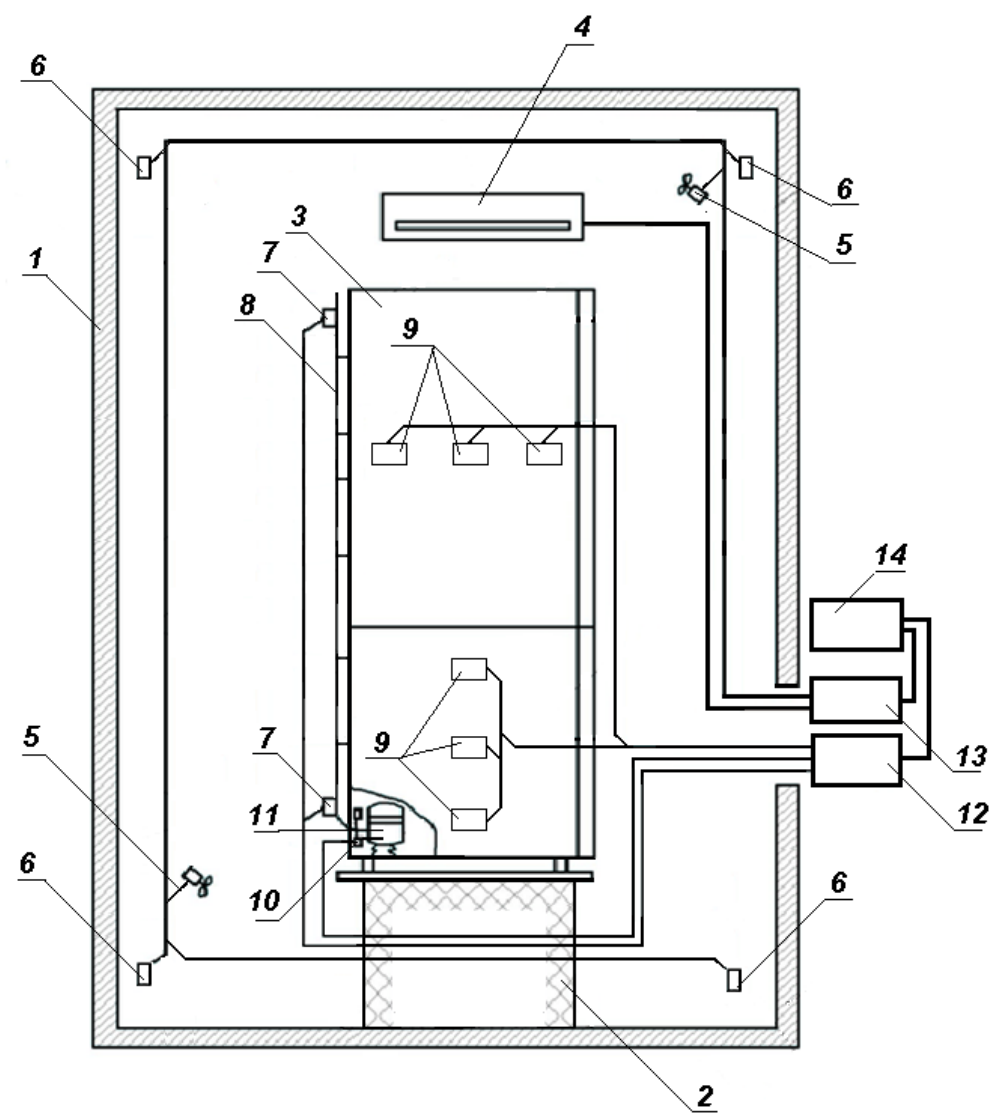

Fig. 2. Scheme of the robotic stand. 1- heat-insulated camera, 2- support, 3- refrigerator, 4 conditioner, 5 -thermal generators, 6temperature sensors, 7 sensors on the condenser, 8 -condenser, 9sensors in cooling cameras, 10- sensor of power consumption, 11-compressor, 12 - block of planning measurements, 13-block of temperature regulations in the heat-insulated camera, 14- controller of management.

Using the button order "Start", the stand is turned on and operates in autonomous mode. In the beginning of the experiment the first value of air temperature t_cam ${ }^{\wedge} 1$ in the heatinsulated camera can be programmatically established, then the processes of tuning and registration in operating modes of the small refrigerating machines are performed followed by measurements and registration of the results of air temperature changes at t_cam ${ }^{\wedge} 1$ in the heat-insulated camera. Results of measurements by means of sensors are recorded in non-volatile memory of the management controller in real time (with the indication of date, time and accompanying records).

Then in the heat-insulated camera the following air temperature indicator $t$ cam^ 2 is automatically established, in accordance to the programmed list of temperatures $\left(\mathrm{t}+\mathrm{cam}^{\wedge} \mathrm{n}\right)$, then tuning and registration at the set operating mode of the refrigerating unit are performed, the measurement, results registration of the second air temperature indicator t_cam ${ }^{\wedge} 2$ are stated in non-volatile memory of the management controller in real time and so on.. till the end of the programmed temperature list $\left(\mathrm{t} \_\right.$cam $\left.{ }^{\wedge} \mathrm{n}\right)$ to perform measurements.

In the block of planned measurements the indicators of temperatures are compared, at least, in one of the SRM cameras for each of the subsequent cycles, till the moment of 
setting standard operating mode, according to the standard [11]. According to a signal from the management controller stating that the operating mode is stable, the sub-program begins registering air temperature indicators of $t_{-} \mathrm{cam}^{\wedge} 1$ in the heat-insulated camera and fill in memory cells all indicators of small refrigerators based on measuring sensors indicators.

Similarly to the described algorithm, the program of the management controller can carry out the installation of other set test conditions, with registration of measurement results for each programmed air temperature indicator $\left(t_{-} \mathrm{cam}^{\wedge} \mathrm{n}\right)$ in the heat-insulated camera. After that, the order "stop" is executed to turn off the stand. The measurement results can be obtained from the controller memory for further processing and analysis, or they can be transmitted via remote communication.

The efficiency of the developed stand, namely the autonomy of its work, is provided due to the block of program temperature changes in the heat-insulated camera. Thus, when the stand is in the automatic mode, test conditions can be programmatically changed without direct participation of the operator. It is important, for example, when researches are carried out at rather high temperatures of $t_{-} \mathrm{cam}^{\wedge} \mathrm{n}(38-430 \mathrm{C})$ in the heat-insulated camera or stability of operation indicators can be taken for $18-23$ hours of continuous operation of the SRM compressor.

The interface and algorithm of measurements of the stand allows to carry out the registration of measurement results in the file forms, with subsequent reading of results using external devices or/and to bring the results on the monitor or the recorder for visualization of measurements results in real time. The stand allows investigating various ways of determining technical condition of household refrigerating appliances $[8,9]$.

The robotic stand is intended for carrying out scientific research, and can be useful to manufacturers of small refrigerators; the research centers engaged in design and research of small refrigerators.

\section{Conclusions}

1. The robotic stand provides the organization of the automated method to carry out researches of the small refrigerator.

2. At the same time the stand provides:

- automatic registration of temperatures in the heat-insulated camera where a research object is observed;

- automatic set of operating mode of small refrigerating machines;

- program temperature changes in the heat-insulated camera, according to the research plan.

3. The developed robotic stand simplifies the process of a research design and modernization of small refrigerators.

\section{References}

1. W.B. Jacobson, Small refrigerator, Moscow: Food industry, p. 162 (1977)

2. Application of RU No. 2005121143 A from 12/2/2003. MPK G01R 31/28. WO 2004/051299(6/17/2004). Diagnostic system for electrical household appliances / MULTIBRAS S.A. Electrodomestikos (BR), (2005)

3. D.A. Lepayev Repair of household refrigerators, M. Legprombytizdat, p.265 (1989)

4. Ampere-second. SU No. 1315762. publ. 08.02.87. The stand for tests of the tight refrigerating unit, (1987) 
5. The patent RU 2525058 C1, MPK F 25b 45/00 ot21.02.2013, publ.10.08.2014, bulletin No. 22. Way of determination of technical condition of the compression household refrigerating appliance (2014).

6. M.A. Lemeshko Way of local determination of technical condition of the compression household refrigerating appliance, etc.//Engineering bulletin of Don.. T. 25. No. 2 (25), pp. 49-54 (2013)

7. Refrigerating household appliances. Characteristics and test methods: GOST P MEK 62552 - 2011. - Introduction. 06 - 06 - 2011. - M.: Standartinform. - 63 p. (2012)

8. M.A. Lemeshko, Innovations in technologies of cultivation of crops: materials of the international scientific and practical conference - Persianovsky st: Don state agrarian university, pp 339-344 ( 2015)

9. Pat. RU No. 2480686, MPK F25B49/02, G01M15/00 from 7/19/2011. Way of determining technical condition of the household refrigerating appliance. 7/19/2011, (2013)

10. A.V. Kozhemyachenko, M.A Lemeshko and etc.//News of higher educational institutions. North Caucasus region. Series: Technical science, No. 4, pp 110-114 (2012)

11.The application for the invention of RU No. 2016144267, from 11/10/2016. The stand for obtaining heat power characteristics of household refrigerating appliances, (2016) 